\title{
Parking functions, tree depth and factorizations of the full cycle into transpositions
}

\author{
John Irving $\|$ and Amarpreet Rattan非 \\ ${ }^{1}$ Department of Mathematics \& Computing Science, St. Mary's University, Halifax, NS, Canada, B3H 3C3 \\ ${ }^{2}$ Department of Economics, Mathematics and Statistics, Birkbeck, University of London, Malet Street, London, WC1E \\ $7 H X, U K$
}

\begin{abstract}
Consider the set $\mathcal{F}_{n}$ of factorizations of the full cycle $(012 \cdots n) \in \mathfrak{S}_{\{0,1, \ldots, n\}}$ into $n$ transpositions. Write any such factorization $\left(a_{1} b_{1}\right) \cdots\left(a_{n} b_{n}\right)$ with all $a_{i}<b_{i}$ to define its lower and upper sequences $\left(a_{1}, \ldots, a_{n}\right)$ and $\left(b_{1}, \ldots, b_{n}\right)$, respectively. Remarkably, any factorization can be uniquely recovered from its lower (or upper) sequence. In fact, Biane (2002) showed that the simple map sending a factorization to its lower sequence is a bijection from $\mathcal{F}_{n}$ to the set $\mathcal{P}_{n}$ of parking functions of length $n$. Reversing this map to recover the factorization (and, hence, upper sequence) corresponding to a given lower sequence is nontrivial.

In this paper we investigate several interesting properties of full cycle factorizations with respect to their upper and lower sequences. In particular, we show that area statistics on upper / lower sequences correspond with inversion / non-inversion statistics on labelled trees. Our study centres around a natural bivariate generalization of the wellstudied tree inversion enumerator of Mallows and Riordan.

Résumé. Considérons l'ensemble des factorisations minimales du cycle complet canonique dans le groupe symétrique sur $n+1$ symboles. En 2002, Biane a trouvé une bijection remarquablement simple de cet ensemble à l'ensemble des fonctions parking de longueur $n$; la bijection mappe une factorisation à la séquence composée de la plus petite élément de chaque transposition. Ainsi, il est absolument trivial à trouver l'image d'un factorisation sous cette fonction, mais inverser cette fonction nécessite beaucoup plus travail. Par ailleurs, en ce qui concerne les fonctions parking, la plus grande élément de chaque transposition semble contenir aucune information. Nous montrons, cependant, que la séquence de la plus grande élément de chaque transposition est intéressante aussi. Notamment, les statistiques d'area naturelle sur cette séquence et la fonction parking correspondent ensemble à deux statistiques naturelles sur les arbres: le nombre inversion (ce qui est bien connu) et le nombre non-inversion. Cela nous permet de présenter une fonction génératrice bivariée, qui est une généralisation naturelle de la fonction génératrice inversion univariée pour les arbres de Mallows et Riordan. Nous donnons aussi quelques résultants alliérs.
\end{abstract}

Keywords. parking functions, tree inversions, minimal factorizations

\footnotetext{
†Email: john.irvingesmu.ca

‡Email: arattan@gmail.com
}

1365-8050 (C) 2016 Discrete Mathematics and Theoretical Computer Science (DMTCS), Nancy, France 


\section{Introduction: Trees and parking functions}

This is an extended abstract, with many proofs omitted. For a more complete exposition, see Irving and Rattan (2016).

Let $\mathcal{T}_{n}$ be the set of labelled rooted trees with vertex set $[n]:=\{0,1, \ldots, n\}$ and root 0 . If $i, j$ are vertices of $T \in \mathcal{T}_{n}$ and $i$ is on the unique path from $j$ to the root, then we say $j$ is an descendant of $i$, or equivalently $i$ is an ancestor of $j$. If $j$ is a descendent of $i$, then the pair $(i, j)$ an inversion of $T$ if $i>j$ and a non-inversion if $j>i$. Let $\operatorname{inv}(T)$ be the number of inversions of the tree $T$ and, for $n \geq 0$, let $I_{n}(q)$ be the inversion enumerator defined by

$$
I_{n}(q)=\sum_{T \in \mathcal{T}_{n}} q^{\operatorname{inv}(T)} .
$$

The first few series for $I_{n}(q)$ are

$$
\begin{aligned}
& I_{0}(q)=1, \\
& I_{1}(q)=1, \\
& I_{2}(q)=2+q, \\
& I_{3}(q)=6+6 q+3 q^{2}+q^{3} .
\end{aligned}
$$

Cayley's famous counting formula for labelled trees on $n$ vertices asserts that $I_{n}(1)=\left|\mathcal{T}_{n}\right|=(n+1)^{n-1}$.

Let $\mathcal{P}_{n}$ be the set of parking functions of length $n$, that is, sequences $\left(a_{1}, a_{2}, \ldots, a_{n}\right) \in[n-1]^{n}$ whose non-decreasing rearrangement $\left(r_{1}, r_{2}, \ldots, r_{n}\right)$ satisfies $r_{i} \leq i-1$. Konheim and Weiss (1966) proved that $\left|\mathcal{P}_{n}\right|=(n+1)^{n-1}$, establishing the henceforth well-studied equality $\left|\mathcal{P}_{n}\right|=\left|\mathcal{T}_{n}\right|$.

It is convenient to view parking functions as labelled Dyck paths, i.e. lattice paths beginning at the origin and remaining weakly below the line $y=x$. One computes the non-decreasing rearrangement $r_{1} \leq r_{2} \leq \cdots \leq r_{n}$ of a given parking function $\left(a_{1}, a_{2}, \ldots, a_{n}\right) \in \mathcal{P}_{n}$, and draws a path whose $j$-th horizontal step is at height $r_{j}$. All horizontal steps at height $j$ are then labelled with the set $\left\{i: a_{i}=j\right\}$, with labels at the same height increasing from left to right.

The area of a parking function $p=\left(a_{1}, \ldots, a_{n}\right)$ is defined by area $(p):=\left(\begin{array}{l}n \\ 2\end{array}\right)-\sum a_{i}$. Equivalently, area $(p)$ is the area between $y=x$ and the Dyck path associated with $p$, not counting the $n$ half-squares along the diagonal. The parking function area enumerators are then defined by

$$
P_{n}(q)=\sum_{p \in \mathcal{P}_{n}} q^{\operatorname{area}(p)},
$$

for $n \geq 1$. The first few $P_{n}(q)$ are easily seen to agree with $I_{n}(q)$. Indeed, it was originally shown by Kreweras (1980) that these two series are equal for all $n$.

Parking functions and tree inversions are connected to a number of other combinatorial objects; see Haglund (2008) for an extensive treatment of the connection between parking functions to representation theory and algebraic geometry, and Gessel (1995, 1980) and Sokal (2009) for the connections between parking functions, tree inversions, the Tutte polynomial and Lagrange inversion.

A variant of parking functions that will prove convenient later in our development are the "suites majeures" of Kreweras (1980). An integer sequence $q=\left(b_{1}, \ldots, b_{n}\right)$ is a major sequence provided its nondecreasing rearragement $\left(r_{1}, \ldots, r_{n}\right)$ satisfies $r_{i} \geq i$ for all $i$, with $r_{n} \leq n$. Clearly $q=\left(b_{1}, \ldots, b_{n}\right)$ 
is a major sequence if and only if its complement $\hat{q}=\left(n-b_{1}, \ldots, n-b_{n}\right)$ is a parking function. We denote the set of all major sequences of length $n$ by $\mathcal{M}_{n}$.

As with parking functions, major sequences of length $n \geq 1$ can be viewed as labelled Dyck paths beginning at the origin and ending at $(n, n)$, while remaining above $y=x$. The area of $q=\left(b_{1}, \ldots, b_{n}\right) \in$ $\mathcal{M}_{n}$ is defined by area $(q):=\sum_{i} b_{i}-\left(\begin{array}{c}n+1 \\ 2\end{array}\right)$, which is again the area (disregarding half-squres) bounded between $y=x$ and the associated path. Note that area $(\hat{q})=\left(\begin{array}{l}n \\ 2\end{array}\right)-\sum_{i}\left(n-b_{i}\right)=\operatorname{area}(q)$, so the area enumerators of parking functions and major sequences coincide.

\section{Refinement of the inversion enumerator}

The inversion enumerator for trees can be refined in the following natural manner. Letting $\operatorname{ninv}(T)$ denote the number of non-inversions in a tree $T \in \mathcal{T}_{n}$, we define

$$
I_{n}(q, t)=\sum_{T \in \mathcal{T}_{n}} q^{\operatorname{inv}(T)} t^{\operatorname{ninv}(T)} .
$$

The first few values of $I_{n}(q, t)$ are shown below:

$$
\begin{aligned}
& I_{0}(q, t)=1 \\
& I_{1}(q, t)=t \\
& I_{2}(q, t)=t^{2}+t^{3}+t^{2} q \\
& I_{3}(q, t)=t^{6}+2 t^{5} q+2 t^{4} q^{2}+t^{3} q^{3}+t^{5}+t^{4} q+t^{3} q^{2}+3 t^{4}+3 t^{3} q+t^{3} .
\end{aligned}
$$

Notice that $\operatorname{ninv}(T)$ is independent from $\operatorname{inv}(T)$ in the sense that trees with the same number of inversions can have a different numbers of non-inversions. However, $\operatorname{ninv}(T)$ and $\operatorname{inv}(T)$ are related via a third tree statistic, which we now define.

Let $\operatorname{dep}(i)$ be the distance from vertex $i$ of $T \in \mathcal{T}_{n}$ to the root. Then the total depth of $T$ is defined by $\operatorname{tdep}(T)=\sum_{i \in T} \operatorname{dep}(i)$. Equivalently, $\operatorname{tdep}(T)$ is simply the number of ordered pairs $(i, j)$ of vertices of $T$ such that $i$ is an ancestor of $j$. Since every such pair is either an inversion or non-inversion of $T$, but not both, we have $\operatorname{tdep}(T)=\operatorname{inv}(T)+\operatorname{ninv}(T)$. Thus

$$
I_{n}(q, q)=\sum_{T \in \mathcal{T}_{n}} q^{\operatorname{tdep}(T)} .
$$

Now consider the exponential generating series

$$
I(q, t, x)=\sum_{n \geq 0} I_{n}(q, t) \frac{x^{n}}{n !}
$$

for our refined inversion enumerators. Upon decomposing a tree into a set of trees by removing its root, and carefully accounting for (non-)inversions, one is led to the functional equation

$$
I(q, t, x)=\exp \left(t \sum_{m=1}^{\infty}\left(t^{m-1}+t^{m-2} q+\cdots+q^{m-1}\right) I_{m-1}(q, t) \frac{x^{m}}{m !}\right) .
$$


We omit the proof of (3), as this is a straightforward generalization of the analogous equation for $I(q, 1, x)$, which has been exposited elsewhere (see Mallows and Riordan (1968) or Gessel (1995), for instance). Note that the asymmetry between $q$ and $t$ is due to trees in $\mathcal{T}_{n}$ always having 0 as a root, so every tree in $\mathcal{T}_{n}$ has at least $n$ non-inversions. Also observe that setting $t=q$ in (3) yields

$$
I(q, q, x)=\exp \left(q \sum_{m=1}^{\infty}\left(m q^{m-1}\right) I_{m-1}(q, q) \frac{x^{m}}{m !}\right)=\exp (q x I(q, q, q x)),
$$

which is immediate from (2) and the exponential formula (see Stanley (1999)).

\section{Minimal factorizations of full cycles into transpositions}

Let $\mathfrak{S}_{[n]}$ denote the symmetric group on the $n+1$ symbols $[n]$, and let $\mathcal{C}_{n}$ denote the subset of $\mathfrak{S}_{[n]}$ consisting of all full cycles (that is, cycles of length $n+1)$. The canonical full cycle $\left(\begin{array}{llll}0 & 1 & \cdots\end{array}\right) \in \mathcal{C}_{n}$ will be specifically denoted by $\sigma_{n}$. We further adopt the conventions that permutations are multiplied left-to-right and transpositions are always written in the form $(a b)$ with $a<b$.

It is well known that any full cycle $\alpha \in \mathcal{C}_{n}$ can be decomposed into $n$ transpositions but no fewer. Accordingly, a sequence $\left(t_{1}, \ldots, t_{n}\right)$ of transpositions satisfying $t_{1} \cdots t_{n}=\alpha$ (or, informally, the expression $t_{1} \cdots t_{n}$ itself) is called a minimal factorization of $\alpha$. We write $\mathcal{F}_{\alpha}$ for the set of such factorizations. For example, we have

$$
\mathcal{F}_{(012)}=\{(01)(02),(02)(12),(12)(01)\} .
$$

Dénes (1959) employed a simple correspondence between minimal factorizations and labelled trees to show that $\left|\mathcal{F}_{\alpha}\right|=\left|\mathcal{T}_{n+1}\right|=(n+1)^{n-1}$ for any $\alpha \in \mathcal{C}_{n}$. Since we also have $\left|\mathcal{P}_{n}\right|=\left|\mathcal{T}_{n+1}\right|$, we anticipate a direct connection between minimal factorizations and parking functions.

To describe the connection, let us first define for any $\alpha \in \mathcal{C}_{n}$ the following functions from $\mathcal{F}_{\alpha}$ to $\mathbb{N}^{n}$ :

$$
\begin{aligned}
& L_{\alpha}:\left(a_{1} b_{1}\right) \cdots\left(a_{n} b_{n}\right) \mapsto\left(a_{1}, \ldots, a_{n}\right) \\
& U_{\alpha}:\left(a_{1} b_{1}\right) \cdots\left(a_{n} b_{n}\right) \mapsto\left(b_{1}, \ldots, b_{n}\right)
\end{aligned}
$$

We refer to $L_{\alpha}(f)$ and $U_{\alpha}(f)$ as the lower and upper sequences of $f \in \mathcal{F}_{\alpha}$, respectively.

In the case $\alpha=\sigma_{n}=\left(\begin{array}{lll}0 & 1 \cdots n\end{array}\right)$, Biane (2002) showed that $L_{\sigma_{n}}$ is a bijection from minimal factorizations of the canonical full cycle to parking functions of length $n$. More generally, it transpires that $L_{\alpha}(f)$ is a parking function and $U_{\alpha}(f)$ a major sequence for all $f \in \mathcal{F}_{\alpha}$, with these mappings being bijective for precisely $2^{n-1}$ full cycles $\alpha \in \mathfrak{S}_{[n]}$. More precisely we have the following result, whose proof we omit in this extended abstract.

Theorem 3.1 Let $\alpha=\left(\begin{array}{ll}0 & i_{1} i_{2} \cdots i_{n}\end{array}\right)$ be any full cycle in $\mathfrak{S}_{[n]}$. For every $f \in \mathcal{F}_{\alpha}$, we have $L_{\alpha}(f) \in \mathcal{P}_{n}$ and $U_{\alpha}(f) \in \mathcal{M}_{n}$. Moreover, $L_{\alpha}$ (respectively, $\left.U_{\alpha}\right)$ is a bijection between $\mathcal{F}_{\alpha}$ and $\mathcal{P}_{n}\left(\right.$ resp. $\mathcal{M}_{n}$ ) if and only if $\left(0, i_{1}, i_{2}, \ldots, i_{n}\right)$ is unimodal. ${ }^{(\mathrm{i})}$

From this point on we shall consider only factorizations of the canonical full cycle $\sigma_{n}=(012 \cdots n)$. As such, we simplify the notation introduced above by writing $\mathcal{F}_{n}$ in place of $\mathcal{F}_{\sigma_{n}}$ and $L(f)$ instead of $L_{\sigma_{n}}(f)$ for $f \in \mathcal{F}_{n}$ (likewise for $U(f)$ ).

(i) Recall that a sequence $\left(c_{1}, \ldots, c_{n}\right)$ is unimodal if $c_{1} \leq c_{2} \leq \cdots \leq c_{i} \geq c_{i+1} \geq \cdots \geq c_{n}$ for some $i$. 


\section{The main result}

Define the lower and upper area of a factorization $f=\left(a_{1} b_{1}\right) \cdots\left(a_{n} b_{n}\right) \in \mathcal{F}_{n}$ by

$$
A_{L}(f)=\left(\begin{array}{l}
n \\
2
\end{array}\right)-\sum_{i=1}^{n} a_{i} \quad \text { and } \quad A_{U}(f)=\sum_{i=1}^{n} b_{i}-\left(\begin{array}{l}
n \\
2
\end{array}\right)
$$

respectively. Written in terms of the area statistic on parking functions and major sequences, we have simply $A_{L}(f)=\operatorname{area}(L(f))$ and $A_{U}(f)=\operatorname{area}(U(f))+n$. Thus the area between the two Dyck paths associated $L(f)$ and $U(f)$, including the $n$ squares along the diagonal, is exactly $A_{L}(f)+A_{U}(f)=$ $\sum_{i}\left(b_{i}-a_{i}\right)$. See Figure 1 for an example.

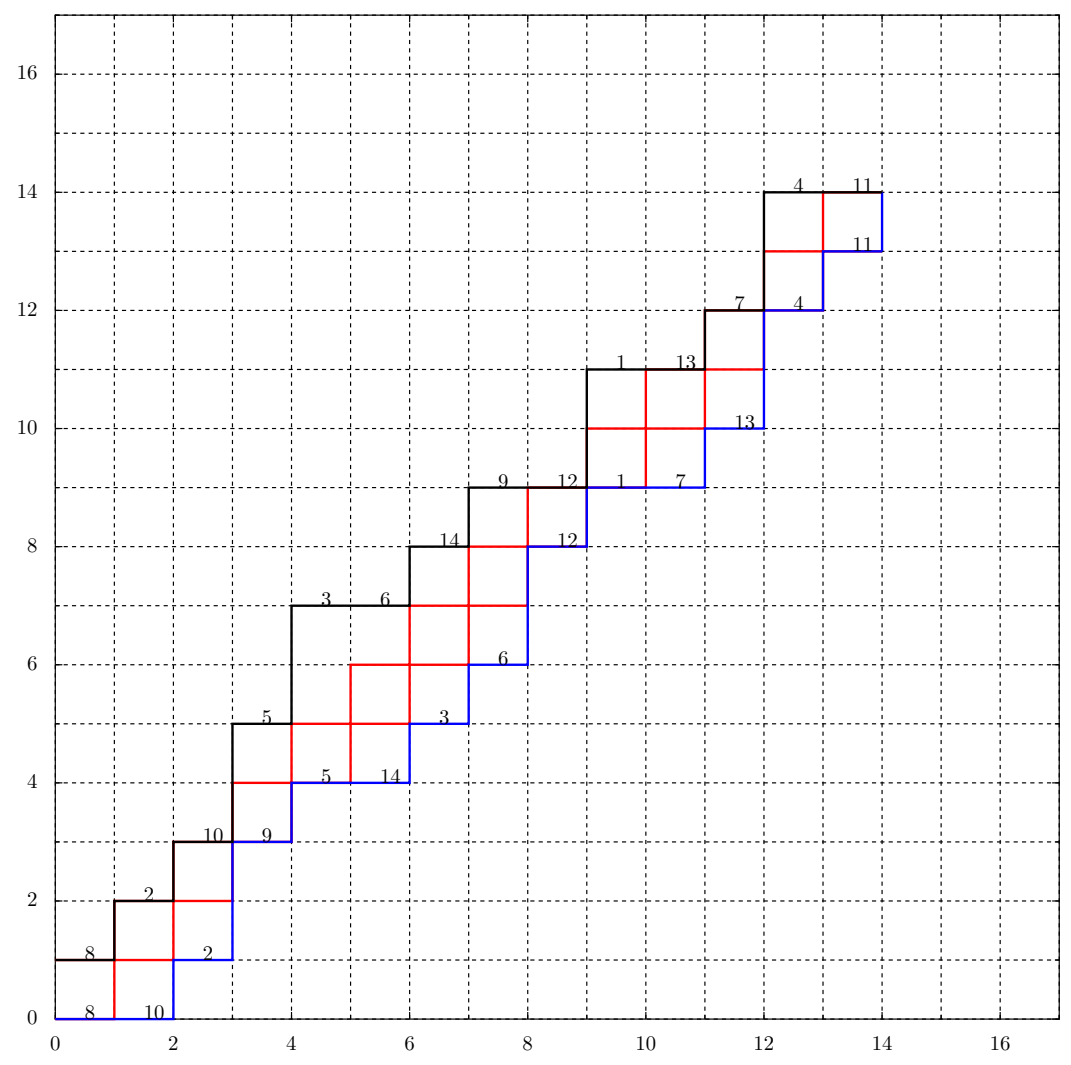

Fig. 1: The factorization $f=(9$ 11)(1 2)(5 7)(12 14)(4 5)(6 7)(9 12)(0 1)(3 9)(0 3)(13 14)(8 9)(10 11)(4 8) $\in$ $\mathcal{F}_{14}$, presented as two labelled Dyck paths. The lower (blue) path corresponds with the parking function $L(f)$ and the upper (black) path with the major sequence $U(f)$. The lower area $A_{L}(f)=7$ is the number of whole squares between the blue path and the line $y=x$. The upper area $A_{U}(f)=22$ consists of the 8 entire squares above the line $y=x$ plus the 14 squares along the diagonal. 
Now define the factorization area enumerators by

$$
F_{n}(q, t)=\sum_{f \in \mathcal{F}_{n}} t^{A_{U}(f)} q^{A_{L}(f)}
$$

for $n \geq 1$ and $F_{0}(q, t)=1$, and let

$$
F(q, t, x)=\sum_{n \geq 0} F_{n}(q, t) \frac{x^{n}}{n !} .
$$

be their generating series. One can quickly verify that the first few values of $F_{n}(q, t)$ are

$$
\begin{aligned}
& F_{0}(q, t)=1 \\
& F_{1}(q, t)=t \\
& F_{2}(q, t)=q t^{2}+t^{3}+t^{2},
\end{aligned}
$$

which agree with the refined tree inversion enumerators $I_{n}(q, t)$. Unsurprisingly, this is not a coincidence:

Theorem 4.1 (Main Theorem) We have

$$
F(q, t, x)=\exp \left(t \sum_{m=1}^{\infty}\left(t^{m-1}+t^{m-2} q+\cdots+q^{m-1}\right) F_{m-1}(q, t) \frac{x^{m}}{m !}\right) .
$$

Comparing with (3), we conclude that $F(q, t, x)$ and $I(q, t, x)$ must coincide, as they agree on the initial terms and satisfy the same recurrence. Thus we have:

Corollary 4.2 The number of trees $T \in \mathcal{T}_{n}$ with $i$ inversions and $j$ non-inversions is equal to the number of factorizations $f \in \mathcal{F}_{n}$ with $A_{L}(f)=i$ and $A_{U}(f)=j$. In particular, the number of trees $T \in \mathcal{T}_{n}$ of total depth $k$ is equal to the number of factorizations $\left(a_{1} b_{1}\right) \cdots\left(a_{n} b_{n}\right) \in F_{n}$ with total difference $\sum_{i}\left(b_{i}-a_{i}\right)=k$.

The proof of Theorem 4.1 comes via a series of lemmas and is outlined in Section 5 , below. An alternative bijective proof will be given in Irving and Rattan (2016).

\section{Proof of the main result}

There are two main stages to the proof of Theorem 4.1. The first is Theorem 5.1, which gives the exponential decomposition of factorizations into connected structures, while preserving the relevant statistics, and the second, given in Theorem 5.5. shows how the connected structures decompose in the necessary way.

Specifically, the relevant connected structures are as follows. Let $\hat{\mathcal{F}}_{n}$ be the subset of $\mathcal{F}_{n}$ whose members contain the transposition $(0 n)$. Set $\hat{\mathcal{F}}_{n}(q, t)$ to be the analogous generating series to the one in (4); that is,

$$
\hat{\mathcal{F}}_{n}(q, t)=\sum_{f \in \hat{F}_{n}} t^{A_{U}(f)} q^{A_{L}(f)} .
$$

Further set $\hat{F}(q, t, x)=\sum_{n \geq 1} \hat{F}_{n}(q, t) \frac{x^{n}}{n !}$. The following theorem brings us part of the way to proving Theorem 4.1 
Theorem 5.1 The generating series $F(q, t, x)$ and $\hat{F}(q, t, x)$ satisfy

$$
F(q, t, x)=\exp (\hat{F}(q, t, x))
$$

Theorem 5.1 follows from Lemma 5.4 below, but we need to show some lemmas first.

Lemma 5.2 If $f \in F_{n}$ then no two transpositions in $f$ cross; that is, $f$ does not have two transpositions $(a b)$ and $(c d)$ with $a \leq c<b \leq d$.

The previous lemma can be seen fairly quickly from the standard presentation of a minimal factorization of $\sigma_{n}$ as trees embedded in the plane, a construction originally due to Moszkowski (1989).

Briefly, every factorization of the full cycle $\left(\begin{array}{llll}0 & 1 & \cdots\end{array}\right)$ can be represented as an edge-labelled verte rooted planar tree, with one distinguished vertex, where the vertices are otherwise unlabelled, and the edge labels around each vertex increase cyclically clockwise with a unique descent. A more thorough description of this is found in Irving (2009) and Bousquet-Mélou and Schaeffer (2000), which gives an account of the more general setting of factorizations of permutations with more than one cycle, or (Irving. 2005. Section 2.4) for an account of the specific case full cycle factorization.

While this construction does not give us the requisite correspondence between factorizations and trees proving Theorem 4.1 or 5.1, it does help us prove the various lemmas we need (namely, Lemmas 5.2, 5.3 and 5.4.

Lemma 5.3 Suppose $f \in \mathcal{F}_{n}$, and let $\left(\begin{array}{ll}0 & c_{1}\end{array}\right),\left(\begin{array}{ll}0 & c_{2}\end{array}\right), \ldots,\left(\begin{array}{ll}0 & c_{r}\end{array}\right)$ be the transpositions containing zero, and appearing in $f$ in that order. Then $c_{1}<c_{2}<\cdots<c_{r}$. Further, suppose that $c_{r}$ appears in transpositions with the elements $d_{1}<d_{2}<\cdots<d_{t}$, and a is the largest index with $d_{a}<c_{r}$. Then $\left(\begin{array}{ll}d_{1} & c_{r}\end{array}\right),\left(\begin{array}{ll}d_{2} & c_{r}\end{array}\right), \ldots,\left(d_{a} c_{r}\right)$ appear in $f$ in that order and all occur after the transposition $\left(0 c_{r}\right)$, and $\left(c_{r} d_{a+1}\right), \ldots,\left(c_{r} d_{t}\right)$ all occur before $\left(\begin{array}{ll}0 & c_{r}\end{array}\right)$ and appear in that order.

Finally, from these two lemmas we obtain the following key result, which provides what is needed to prove Theorem 5.1 .

Lemma 5.4 Let $n>1$ and $f=\left(\left(a_{1} b_{1}\right), \ldots,\left(a_{n} b_{n}\right)\right) \in \mathcal{F}_{n}$. Then there is some (non-empty) sequence of indices $1 \leq i_{1}<i_{2}<\cdots<i_{k} \leq n$ with the following properties:

1. $a_{i_{k}}=0, b_{i_{1}}=n$ and $b_{i_{j}}=a_{i_{j-1}}$, for $2 \leq j \leq k$, and for every other transposition $\left(a_{j} b_{j}\right) \in f$ there is an index $1 \leq t \leq k$ such that

$$
a_{i_{t}} \leq a_{j}<b_{j} \leq b_{i_{t}}
$$

2. for each $1 \leq t \leq k$ there are $b_{i_{t}}-a_{i_{t}}$ transpositions $\left(a_{j} b_{j}\right)$ (including $\left(a_{i_{t}} b_{i_{t}}\right)$ itself) in $f$ satisfying Condition (1);

3. for each $1 \leq t \leq k$ the product of all transpositions satisfying Condition (2), including $\left(a_{i_{t}} b_{i_{t}}\right)$, in the stated order of the factorization is $\left(a_{i_{t}} a_{i_{t}}+1 \cdots b_{i_{t}}\right)$. 
We will not give a proof of Lemma 5.4 in this abstract, but we will give an example illustrating the key concepts of Lemmas 5.3 and 5.4 . Consider the following member $f \in \mathcal{F}_{14}$ :

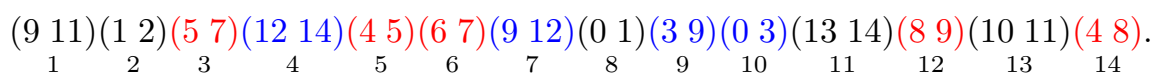

Two transpositions contain the symbol 0 and they appear in the order stated in Lemma 5.3 Furthermore, the indices $\left(i_{1}, \ldots, i_{k}\right)$ in Lemma 5.41 1 are $(4,7,9,10)$, or are the transpositions given in blue. Focussing on the transposition (39), according to Lemma 5.42 2 there are five other transpositions in the factorization whose elements are contained between 3 and 9. Those transpositions are in positions 3, 5, 6, 12, 14 (the red transpositions). The product of these transpositions (including the transposition (39)) in the order given is $(34 \cdots 9)$, as claimed in Lemma 5.4 3 Notice that this is also the example given in Figure 1 . The condition in Lemma 5.4/3 has a nice graphical interpretation when looking at the two lattice paths given by the lower and upper sequences of a factorization. Looking at Figure 1, the transpositions 3, 5, 6, 9, 12, 14 correspond to a region fully enclosed by the two lattice paths. That is, the upper and lower paths meet at the endpoints given by the coordinates $(3,3)$ and $(9,9)$.

Thus, in light of Theorem 5.1, what remains is to show the following theorem.

Theorem 5.5 The generating series $\hat{F}(q, t, x)$ satisfies

$$
\hat{F}(q, t, x)=t \sum_{m=1}^{\infty}\left(t^{m-1}+t^{m-2} q+\cdots+q^{m-1}\right) F_{m-1}(q, t) \frac{x^{m}}{m !} .
$$

To prove Theorem 5.5, it suffices to show there exists a bijection

$$
\Gamma: \hat{\mathcal{F}}_{n} \rightarrow\left\{\left(k, \mathcal{F}_{n-1}\right): k \in[n-1]\right\}
$$

where for $f \in \hat{\mathcal{F}}_{n}$ we have $A_{U}(f)=A_{U}(\Gamma(f))+k+1$ and $A_{L}(f)=A_{L}(\Gamma(f))+n-k-$ 1. Thus, the contributions of $f$ and $\Gamma(f)$ to their respective generating series are $t^{A_{U}(f)} q^{A_{L}(f)}$ and $\left(t^{k+1} q^{n-k-1}\right) t^{A_{U}(\Gamma(f))} q^{A_{L}(\Gamma(f))}$.

Note one consequence of the existence of a bijection (5): the number of minimal factorizations of $\sigma_{n}$ with $(0 n)$ as a transposition is $n^{n-1}$.

We first need a lemma.

Lemma 5.6 Suppose that $f=\left(\alpha_{1}, \ldots, \alpha_{n}\right) \in \hat{\mathcal{F}}_{n}$. Then there is a unique index $k$ such that $\alpha_{k}=$ $(0 n)$. Furthermore, for every $i<k$, no transposition $\alpha_{i}$ contains the symbol $n$ and for every $i>k$ no transposition $\alpha_{i}$ contains the symbol 0 .

The proof of Lemma 5.6 is relatively simple. The uniqueness of $k$ is clear as the factorization of $\sigma_{n}$ would otherwise fail to be minimal, as argued in at the beginning of Section 3 . The second and third claims are covered by Lemma 5.3 with $c_{r}=n$ there.

Thus, if we define $\mathcal{F}_{n, k}$ as the set of $f \in \hat{\mathcal{F}}_{n}$ with $(0 n)$ as the $k^{\text {th }}$ transposition, we see that $\hat{\mathcal{F}}_{n}=$ $\dot{\cup}_{1 \leq k \leq n} \hat{\mathcal{F}}_{n, k}$.

Define a function $\phi: \hat{\mathcal{F}}_{n} \rightarrow \hat{\mathcal{F}}_{n}$ by the following rules. Set $f=\left(\alpha_{1}, \ldots, \alpha_{n}\right) \in \hat{\mathcal{F}}_{n}$. If $f \in \hat{\mathcal{F}}_{n, k}$ for $k \leq n-1$, then define

$$
\phi(f)=\left(\bar{\alpha}_{n}, \alpha_{1}, \ldots, \alpha_{n-1}\right)
$$


where $\bar{\alpha}_{n}=(i-1 j-1)$ if $\alpha_{n}=(i j)$. Notice from Lemma 5.6 that $i \neq 0$, so $i-1 \geq 0$. If $f \in \hat{\mathcal{F}}_{n, n}$ then $\alpha_{n}=(0 n)$ and define

$$
\phi(f)=\left(\alpha_{n}, \bar{\alpha}_{1}, \ldots, \bar{\alpha}_{n-1}\right)
$$

where $\bar{\alpha}_{k}=(i+1 j+1)$ if $\alpha_{k}=(i j)$ for $1 \leq k \leq n-1$. By Lemma 5.6 for each $\alpha_{k}=(i j)$ with $1 \leq k \leq n-1$, we see that $j \neq n$, so $j+1 \leq n$. We call $\phi$ the forwards rotation operator.

Though the forwards rotation operator clearly cyclically rotates the element $(0 n)$ to the right, it is of course necessary to verify that it preserves factorizations of the $\sigma_{n}$. After verifying that the forwards rotation operation is well-defined, it's not difficult to see that it has a well-defined inverse $\beta$, the backwards rotation operator. The details proving the above properties of the rotation operators are omitted here. Whence we obtain the following lemma.

Lemma 5.7 The functions $\phi$ and $\beta$ are well-defined functions and are both bijective from $\hat{\mathcal{F}}_{n, k}$ to $\hat{\mathcal{F}}_{n, k+1}$ (where $\hat{\mathcal{F}}_{n, k+1}$ is defined as $\hat{\mathcal{F}}_{n, 1}$ for $k=n$ ) for all $1 \leq k \leq n$.

The following example shows the forwards rotation operator working on a factorization. Let $f=$ (1 2), (l 1 1), (l $\left.\begin{array}{ll}0 & 3\end{array}\right),\left(\begin{array}{ll}0 & 4\end{array}\right) \in \hat{\mathcal{F}}_{4,4}$. Then

$$
\begin{aligned}
& \phi(f)=\left(\begin{array}{ll}
0 & 4
\end{array}\right),(2 \text { 3), (1 2), (1 4) } \\
& \phi^{2}(f)=\left(\begin{array}{ll}
0 & 3
\end{array}\right),(04),(23),(12) \\
& \phi^{3}(f)=\left(\begin{array}{lll}
0 & 1
\end{array}\right),\left(\begin{array}{lll}
0 & 3
\end{array}\right),\left(\begin{array}{ll}
0 & 4
\end{array}\right),\left(\begin{array}{ll}
2 & 3
\end{array}\right) \\
& \phi^{4}(f)=\left(\begin{array}{ll}
1 & 2
\end{array}\right),\left(\begin{array}{ll}
0 & 1
\end{array}\right),\left(\begin{array}{ll}
0 & 3
\end{array}\right),\left(\begin{array}{ll}
0 & 4
\end{array}\right) .
\end{aligned}
$$

Now define a function $\gamma: \hat{\mathcal{F}}_{n, n} \rightarrow \mathcal{F}_{n-1}$ by $\gamma\left(\left(\alpha_{1}, \ldots, \alpha_{n}\right)\right)=\left(\alpha_{1}, \ldots, \alpha_{n-1}\right)$. The function $\gamma$ is clearly bijective and well-defined. Finally define $\Gamma: \hat{\mathcal{F}}_{n} \rightarrow\left\{\left(k, \mathcal{F}_{n-1}\right): k \in[n-1]\right\}$ by if $f \in \hat{F}_{n, n-k}$ then $\Gamma(f)=\left(k, \gamma \circ \phi^{k}(f)\right)$. It follows the function $\Gamma$ is a bijection from $\hat{\mathcal{F}}_{n} \rightarrow\left\{\left(k, \mathcal{F}_{n-1}\right): k \in[n-1]\right\}$.

Given a factorization $f \in \hat{F}_{n}$, it is a straightforward exercise to relate the upper and lower areas of both $\phi(f)$ and $\gamma(f)$ to the upper and lower areas of $f$. Thus, for any $f \in \hat{\mathcal{F}}_{n, n-k}$ it follows that

$$
A_{U}(\Gamma(f))=A_{U}(f)-k-1 \quad \text { and } \quad A_{L}(\Gamma(f))=A_{L}(f)+k-n+1,
$$

showing that the function $\Gamma$ has the requisite properties to satisfy the conditions in 5 .

\section{Generalizations}

For a labelled tree $T$ and a pair $(i, j)$ of nonnegative integers, let $H_{i, j}(T)$ denote the number of vertices $v \in T$ which have $i$ descendants smaller than $v$ and $j$ descendants larger than $v$. Up to now we have been concerned with the distribution of the pair

$$
(\operatorname{inv}(T), \operatorname{ninv}(T))=\sum_{i, j}(i, j) H_{i, j}(T)
$$

and its relation to natural statistics on parking functions and minimal factorizations. More generally, it is fruitful to investigate the full matrix statistic $\mathbf{H}(T)=\left[H_{i, j}(T)\right]$ and its analogues for other "tree-like" objects (i.e. combinatorial objects known to be equinumerous with labelled trees). Progress along these lines is detailed in Irving and Rattan (2016). Here we give only a somewhat specialized overview. 
First consider the vector $\operatorname{inv}(T)=\left(\sum_{j} H_{i, j}(T)\right)_{i \geq 0}$ of row sums of $\mathbf{H}(T)$. It is known that the distribution of $\operatorname{inv}(T)$ over trees coincides with the distribution of a natural statistic on parking functions. In particular, Shin (2008) defines the jump statistic on parking functions and gives a simple bijection $\zeta$ : $\mathcal{T}_{n} \rightarrow \mathcal{P}_{n}$ satisfying $\operatorname{inv}(T)=\operatorname{jump}(\zeta(T))$. Note that the sum of the entries of $\operatorname{inv}(T)$ is $\operatorname{simply} \operatorname{inv}(T)$, and it is clear from the definition of $\mathbf{j u m p}(p)$ that its entries sum to area $(p)$. One can similarly consider the vector $\operatorname{ninv}(T)$ of column sums of $\mathbf{H}(T)$, and it can be shown that the joint statistic $(\operatorname{inv}(T), \operatorname{ninv}(T))$ over all trees $T \in \mathcal{T}_{n}$ is equidistributed with a natural refinement of $\left(A_{L}(f), A_{U}(f)\right)$ over all factorizations $f \in \mathcal{F}_{n}$.

Next consider the vector $\operatorname{hook}(T)=\left(\operatorname{hook}_{k}(T)\right)_{k \geq 0}$ consisting of the antidiagonal sums hook $k(T):=$ $\sum_{i+j=k} H_{i, j}(T)$. Note that hook $k(T)$ is the number of vertices of $T$ having exactly $k$ descendants (that is, having hook length $k$ ) and the total depth of $T$ is therefore $\operatorname{tdep}(T)=\sum_{k} k \cdot \operatorname{hook}_{k}(T)$. As indicated in Corollary 4.2, the distribution of total depth over labelled trees matches that of total difference over minimal factorizations. More generally, it transpires that the distribution of $\operatorname{hook}(T)$ for $T \in \mathcal{T}_{n}$ matches that of the difference index $\operatorname{diff}(f)$ for $f \in \mathcal{F}_{n}$, where the $k$-th entry of $\operatorname{diff}(f)$ is the number of factors $(a b)$ of $f$ having $b-a=k$. A similar result appears in Goulden and Yong (2002), although there the authors compute transposition differences circularly, replacing $b-a$ with $\min \{b-a, n+1+a-b\}$, which has the effect of disguising the connection with hook lengths. It is interesting to note that hook length formulae for labelled trees due to Chen et al. (2009) immediately yield the curious identities

$$
\sum_{f \in \mathcal{F}_{n}} \prod_{i} \frac{1}{b_{i}-a_{i}}=n ! \quad \text { and } \quad \sum_{f \in \mathcal{F}_{n}} \prod_{i} \frac{1}{\left(b_{i}-a_{i}\right)^{2}}=\frac{(n+2) !}{2^{n+1}}
$$

where both sums extend over all factorizations $f=\left(a_{1} b_{1}\right) \cdots\left(a_{n} b_{n}\right) \in \mathcal{F}_{n}$.

It is natural to investigate analogues of hook length and total depth for other tree-like objects. For parking functions, the analogue of hook length is somewhat convoluted and we do not describe it here. Instead we discuss a more specialized statistic that mates with total depth.

Let $p=\left(a_{1}, \ldots, a_{n}\right)$ be a parking function and consider its associated labelled Dyck path $P$. Imagine a ball starting at the point $(0,0)$ and moving east until it encounters a vertical step of $P$, at which time it "bounces" north and continues until it encounters the line $y=x$, where it "bounces" east until it hits $P$, etc. The ball continues to bounce between $P$ and $y=x$ until it stops at $(n, n)$. If $0=i_{1}<\cdots<i_{k}=n$ are the (integer) $x$-coordinates where the ball intersects $y=x$, then we set

$$
\operatorname{bounce}(p)=\sum_{j}\left(n-i_{j}\right)
$$

For example, the parking function $p=(9,1,5,12,4,6,9,0,3,0,13,8,10,4)$ corresponds with the lower path in Figure 1 The bounce indices $\left(i_{1}, \ldots, i_{k}\right)$ are $(0,2,3,4,6,8,9,11,12,13,14)$, giving bounce $(p)=$ $14+12+11+10+8+6+5+3+2+1+0=72$.

Computing the bounce of small parking functions suggests the following theorem, which indeed holds true in general. We omit the proof here.

Theorem 6.1 The following objects are equinumerous: (1) parking functions $p \in \mathcal{P}_{n}$ with bounce $(p)=$ $k$, (2) trees $T \in \mathcal{T}_{n}$ of total depth $k$, and (3) minimal factorizations $\left(a_{1} b_{1}\right) \cdots\left(a_{n} b_{n}\right) \in \mathcal{F}_{n}$ with total difference $\sum_{i}\left(b_{i}-a_{i}\right)=k$. 
Bounce was originally defined by Haglund Haglund (2003) to provide a combinatorial interpretation of the $(q, t)$-Catalan numbers and the Hilbert series of the space of diagonal harmonics. An extensive treatment of this connection can be found in Haglund (2008). Interestingly, it is clear from algebraic considerations that the joint distribution of bounce and area is symmetric over Dyck paths of a given length; however, no combinatorial proof of this symmetry is known.

Finally, we note that a number of the above results can be generalized to obtain analogous correspondences between $k$-cacti, $k$-parking functions (see Yan (1997)), and minimal factorizations of a full cycle into cycles of length $k$.

\section{References}

P. Biane. Parking functions of types A and B. Electron. J. Combin., 9, 2002.

M. Bousquet-Mélou and G. Schaeffer. Enumeration of planar constellations. Adv. in Appl. Math., pages 337-368, 2000.

W. Y. C. Chen, O. X. Q. Gao, and P. L. Guo. Hook length formulas for trees by Han's expansion. arXiv:math.CO/0903.3342, 2009.

J. Dénes. The representation of a permutation as the product of a minimal number of transpositions and its connection with the theory of graphs. Publ. Math. Inst. Hungar. Acad. Sci., 4:63-70, 1959.

I. Gessel. A noncommutative generalization and $q$-analog of the lagrange inversion formula. Trans. Amer. Math. Soc., pages 455-482, 1980.

I. Gessel and B. Sagan. The tutte polynomial of a graph, depth-first search, and simplicial complex partitions. Electronic J. Combinatorics, 3(2), 1996.

I. M. Gessel. Enumerative applications of a decomposition for graphs and digraphs. Discrete Math., pages 257-271, 1995.

I. Goulden and D. M. Jackson. Transitive factorisations into transpositions and holomorphic mappings on the sphere. Proc. AMS, 125(1):51-60, January 1997.

I. P. Goulden and D. M. Jackson. The combinatorial relationship between trees, cacti and certain connection coefficients for the symmetric group. European J. Combin., 13(5):357-365, 1992. ISSN 01956698 .

I. P. Goulden and A. Yong. Tree-like properties of factorizations. J. Combin. Theory. Ser. A, 98:106-117, 2002.

J. Haglund. Conjectured statistics for the $q, t$-Catalan numbers. Adv. Math., 175(2), 2003.

J. Haglund. The q,t-Catalan Numbers and the Space of Diagonal Harmonics: With an Appendix on the Combinatorics of Macdonald Polynomials, volume 41 of University Lecture Series. American Mathematical Society, 2008.

J. Irving. Combinatorial Constructions for Transitive Factorizations in the Symmetric Group. PhD thesis, University of Waterloo, 2005. 
J. Irving. Minimal transitive factorizations of permutations into cycles. Canad. J. Math., pages 1092$1117,2009$.

J. Irving and A. Rattan. Factorizations of the full cycle into transpositions, tree inversions and tree depth. forthcoming, 2016.

A. G. Konheim and B. Weiss. An occupancy discipline and applications. SIAM J. Applied Math., 14: 1266-1274, 1966.

G. Kreweras. Une famille de polynômes ayant plusiers propriétés énumeratives. Periodica Math. Hung., 11:309-320, 1980.

C. Mallows and J. Riordan. The inversion enumerator for labeled trees. Bull. Amer. Math. Soc., 74:92-94, 1968.

P. Moszkowski. A solution to a problem of dénes: a bijection between trees and factorizations of cyclic permutations. European J. Combinatorics, 10:13-16, 1989.

H. Shin. A new bijection between forests and parking functions. arXiv:math.CO/0810.0427, 2008.

A. Sokal. A ridiculously simple and explicit implicit function theorem. arXiv:math-cv/0902.0069, 2009.

R. P. Stanley. Enumerative Combinatorics, volume 2. Cambridge University Press, 1999.

C. H. Yan. Generalized tree inversions and k-parking functions. J. Combin. Theory. Ser. A, 79:268-280, 1997.

C. H. Yan. Generalized parking functions, tree inversions and multicolored graphs. Adv. Appl. Math., 27: 641-670, 2001. 